\title{
Stress-induced cardiomyopathy after negative pressure pulmonary edema during emergence from anesthesia -A case report-
}

\author{
Su Hyun Lee ${ }^{1,2}$, Chul Ho Chang ${ }^{1,2}$, Jeong Soo Park ${ }^{1}$, and Sang Beom Nam ${ }^{1,2}$ \\ ${ }^{1}$ Department of Anesthesiology and Pain Medicine, ${ }^{2}$ Anesthesia and Pain Research Institute, Yonsei University College of Medicine, \\ Seoul, Korea
}

Stress-induced cardiomyopathy (SICM) presenting as an acute myocardial dysfunction is characterized by transient left ventricular wall motion abnormality, which has been known to be associated with excessive catecholamine production caused due to various types of stress. Sympathetic hyperactivity is common during the perioperative period, and reports of SICM occurring during this period have actually increased. We present a case of SICM following negative pressure pulmonary edema due to upper airway obstruction during emergence from anesthesia. Excessive catecholamine release in response to respiratory difficulty could have been the underlying inciting factor. (Korean J Anesthesiol 2012; 62: 79-82)

Key Words: Negative pressure pulmonary edema, Stress-induced cardiomyopathy.

Stress-induced cardiomyopathy (SICM) presenting as an acute myocardial dysfunction is characterized by transient left ventricular wall motion abnormality, which has been known to be associated with excessive catecholamine production that is precipitated by physical or emotional stress, but its pathophysiology has still not been fully understood [1]. Various procedures including surgery are also the principal relevant factors. Sympathetic hyperactivity is common during the perioperative period, and reports of SICM occurring during this period have actually increased [2-12]. Therefore anesthesiologists should pay attention to this clinical syndrome, however the exact pathophysiologic mechanism or prevalence is not well known.

We present a case of SICM following negative pressure pulmonary edema (NPPE) due to upper airway obstruction during emergence from anesthesia.

\section{Case Report}

A 56-year-old man, $172 \mathrm{~cm}$ in height and $85 \mathrm{~kg}$ in weight, was scheduled for tonsillectomy to rule out tonsillar cancer. He had a medical history of hypertension and non-insulin dependent

Received: April 22, 2011. Revised: 1st, June 3, 2011; 2nd, June 22, 2011. Accepted: June 29, 2011.

Corresponding author: Sang Beom Nam, M.D., Department of Anesthesiology and Pain Medicine, Gangnam Severance Hospital, 211, Unjuro, Gangnam-gu, Seoul 135-720, Korea. Tel: 82-2-2019-6082, Fax: 82-2-3463-0940, E-mail: sbnam@yuhs.ac

(c) This is an open-access article distributed under the terms of the Creative Commons Attribution Non-Commercial License (http:// creativecommons.org/licenses/by-nc/3.0/), which permits unrestricted non-commercial use, distribution, and reproduction in any medium, provided the original work is properly cited. 
diabetes since the last 5 years and he was taking the following medications; Valsartan $\left(\right.$ Diovan $\left.^{\circledR}\right)$, Amlodipine + Atorvastatin $\left(\right.$ Caduet $^{\circledR}$ ), Sulodexide (Vessel Due $\mathrm{F}^{\circledR}$ ), Fenofibrate (Lipidil Supra ${ }^{\circledR}$ ), Aspirin $\left(\right.$ Astrix $^{\circledR}$ ), Pregabalin (Lyrica ${ }^{\circledR}$ ) and Glibenclamide + Metformin $\left(\right.$ Gluriad $\left.^{\circledR}\right)$. During endocrinology clinic follow-up, chest X-ray showed three small well-defined nodular opacities in the left lower lung field and high-resolution computed tomography (CT) of chest showed suspicious metastatic nodules in lower lobes of both lungs and a small nodular lesion with high attenuation in the ampulla of Vater. To detect the original cancer lesion, esophagogastroduodenoscopy was performed, which showed no specific findings except gastric erosion and duodenal ulcer. In addition, abdominal ultrasound, colonoscopy, and positron emission tomographycomputed tomography were performed, but the original cancer lesion could not be detected. However, chest CT for CT-guided needle aspiration biopsy showed lymph node enlargement in the left oropharynx which could be the suspected primary focus, and hence a tonsillectomy was scheduled.

Preoperative laboratory tests were all within normal limits. The electrocardiogram (EKG) showed nonspecific ST abnormality with normal sinus rhythm at a rate of 93 beats/min and a transthoracic echocardiogram showed normal sized cardiac chambers and normal global left ventricular (LV) systolic function with an ejection fraction (EF) of 58\%. A treadmill exercise stress test showed equivocal changes in stage 4 . His functional capacity was more than 4 METs and he had no specific symptoms, but he had a lot of concerns about the progress and prognosis of pulmonary nodules that might be cancer metastasis. He denied any symptoms of chest pain or dyspnea preoperatively.

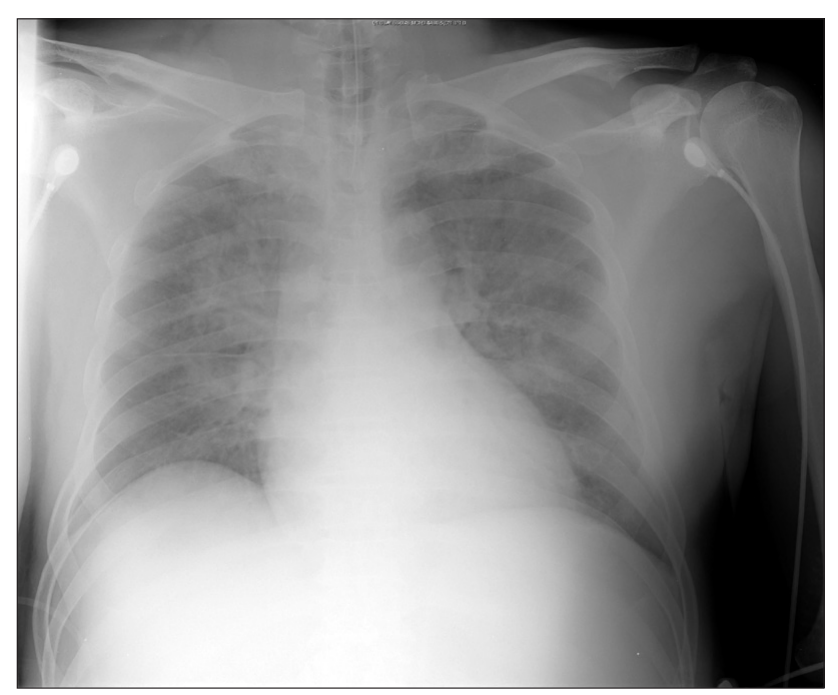

Fig. 1. Immediate postoperative chest AP radiograph shows increased opacity in bilateral perihilar areas.
The patient was premedicated with intravenous glycopyrrolate $0.2 \mathrm{mg}$. Before anesthesia induction, his blood pressure was 165/97 mmHg, heart rate (HR) was 76 beats/min and peripheral oxygen saturation $\left(\mathrm{SpO}_{2}\right)$ was $97 \%$. Anesthesia was induced with propofol $120 \mathrm{mg}$ and continuous infusion of remifentanil. After neuromuscular block was achieved with $50 \mathrm{mg}$ rocuronium, he was intubated with an $8.0 \mathrm{~mm}$ inner diameter endotracheal tube. Anesthesia was maintained with sevoflurane in $60 \%$ oxygen with air and systolic blood pressure was maintained at $100-160 \mathrm{mmHg}$, diastolic blood pressure at $65-100 \mathrm{mmHg}$, $\mathrm{HR}$ at $80-90$ beats/min, and $\mathrm{SpO}_{2}$ above $98 \%$. Tonsillectomy was performed and the surgery lasted for 25 minutes. At the conclusion of tonsillectomy, suction catheter was applied to the endotracheal tube and oral cavity, and there was a little secretion or blood. During emergence from anesthesia, $100 \%$ oxygen was given and when the patient started to breathe spontaneously, muscle relaxant was reversed with neostigmine $1.0 \mathrm{mg}$ and glycopyrrolate $0.2 \mathrm{mg}$. Extubation was performed after he could obey verbal commands such as opening the eyes. Immediately after extubation, the patient had difficulty in breathing and developed inspiratory stridor suggesting upper airway obstruction. An oral airway was inserted and 100\% oxygen was administered via a face mask during spontaneous assisted ventilation. However, he continued to have difficulty in breathing and $\mathrm{SpO}_{2}$ decreased to $77 \%$, blood pressure was 164/101 mmHg, and HR was 125 beats/min. Reintubation was performed approximately 5 minutes after extubation. After reintubation, a large amount of pinkish, frothy secretion was suctioned from the endotracheal tube, arterial blood gas analysis (ABGA) showed a pH of 7.24, arterial carbon dioxide tension $\left(\mathrm{PaCO}_{2}\right)$ of $55.6 \mathrm{mmHg}$, arterial oxygen tension $\left(\mathrm{PaO}_{2}\right)$ of $47.9 \mathrm{mmHg}$ and arterial oxygen saturation $\left(\mathrm{SaO}_{2}\right)$ of $74.1 \%$ at an $\mathrm{FiO}_{2}$ of 1.0 and chest X-ray showed increased opacity in bilateral perihilar areas (Fig. 1), consistent with NPPE. With endotracheal suctioning and administration of furosemide 10 mg, ABGA showed a pH of 7.23, $\mathrm{PaCO}_{2}$ of $53.7 \mathrm{mmHg}, \mathrm{PaO}_{2}$ of $75.7 \mathrm{mmHg}$ and $\mathrm{SaO}_{2}$ of $91.9 \%$ at an $\mathrm{FiO}_{2}$ of 0.8 and positive end-expiratory pressure (PEEP) of $10 \mathrm{cmH}_{2} 0$. The patient was transferred to the intensive care unit (ICU).

After admission to ICU, mechanical ventilation was maintained at an $\mathrm{FiO}_{2}$ of 0.8 and PEEP of $12 \mathrm{cmH}_{2} \mathrm{O}$, and $\mathrm{SpO}_{2}$ increased to $96 \%$. Because blood pressure was $77 / 51 \mathrm{mmHg}$ and HR 106 beats/min, a dopamine infusion was initiated. However, blood pressure did not increase, and a norepinephrine infusion was initiated, blood pressure was maintained at around 120/70 mmHg.

After approximately 5 hours of reintubation, EKG changes were seen on the bedside monitor. The 12-lead EKG revealed STsegment elevation in leads $\mathrm{V}_{2-4}$ and $\mathrm{T}$-wave inversion in leads I, aVL, $V_{2-6}$ (Fig. 2). Cardiac biomarker assay showed an increased 
CK-MB level of $8.8 \mathrm{ug} / \mathrm{L}$ and an increased troponin I level of $2.03 \mathrm{ug} / \mathrm{L}$. Bedside echocardiography and emergency coronary angiography (CAG) were performed. The echocardiogram revealed akinesia of the left ventricular wall from mid to apex causing apical ballooning (Fig. 3) and a reduced EF of $47 \%$. The CAG showed minimal luminal narrowing and apical ballooning was shown on the left ventriculogram. The patient was diagnosed with SICM.

On postoperative day (POD) 1, ABGA at an $\mathrm{FiO}_{2}$ of 0.4 and PEEP of $10 \mathrm{cmH}_{2} \mathrm{O}$ showed a pH of $7.39, \mathrm{PaCO}_{2}$ of $42.5 \mathrm{mmHg}$, $\mathrm{PaO}_{2}$ of $132.9 \mathrm{mmHg}$ and $\mathrm{SaO}_{2}$ of $99.0 \%$. Although chest X-ray appeared to have improved, blood pressure was $82 / 56 \mathrm{mmHg}$ and HR was 114 beats/min, and a dobutamine infusion was added.

On POD 2, both norepinephrine and dopamine infusions were tapered off. On POD 4, chest X-ray showed more improvement, he did not have difficulty in spontaneous breathing and ABGA at an $\mathrm{FiO}_{2}$ of 0.4 and PEEP of $5 \mathrm{cmH}_{2} \mathrm{O}$ showed a pH of 7.45, $\mathrm{PaCO}_{2}$ of $37.9 \mathrm{mmHg}, \mathrm{PaO}_{2}$ of $106.9 \mathrm{mmHg}$ and $\mathrm{SaO}_{2}$ of $99.8 \%$, and hence he has weaned from mechanical ventilation. On POD 5, dobutamine infusion was tapered off, a follow-up echocardiogram revealed no regional wall motion abnormality with an $\mathrm{EF}$ of $60 \%$, and he was transferred to the general ward and was discharged on POD 8 without any complications.

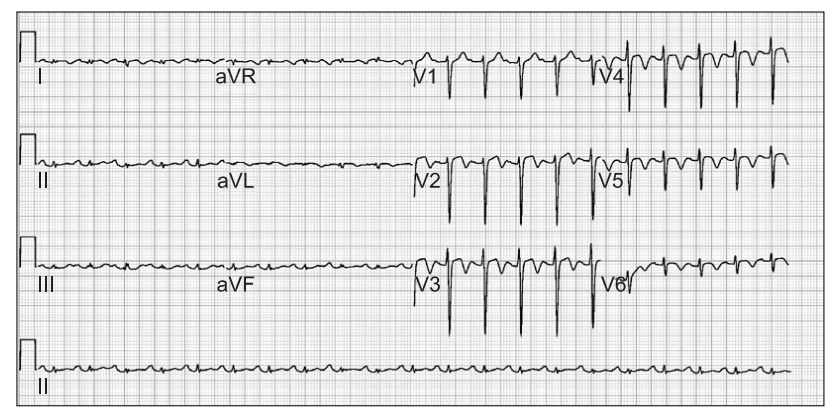

Fig. 2. Postoperative electrocardiogram shows ST segment elevation in leads $\mathrm{V}_{2-4}$ and T-wave inversion in leads $\mathrm{I}$, aVL, $\mathrm{V}_{2-6}$.

\section{Discussion}

Since SICM was first described as Takotsubo-type cardiomyopathy by Sato et al. in 1990 [13], it has been referred to by various names according to the characteristic clinical features [1]. The occurrence of transient left ventricular wall motion abnormality, the so-called apical ballooning, in the absence of epicardial coronary artery obstruction, is a unique clinical feature, but recently various cases presenting other patterns of wall motion abnormality have been reported. Bybee and Prasad [14] proposed the following diagnostic criteria: 1) transient LV wall motion abnormalities involving the apical and/or midventricular segments with wall motion abnormalities extending beyond a single epicardial coronary distribution, 2) the absence of obstructive epicardial coronary artery disease or angiographic evidence of acute plaque rupture that could be responsible for the observed wall motion abnormality, 3) troponin elevation or new EKG abnormalities such as transient ST segment elevation and/or diffuse T-wave inversions. However, recently some case reports have described that some patients seemed to have SICM, but their clinical features did not correspond to the above mentioned criteria, and therefore a new diagnostic approach is needed.

The diagnosis of SICM in this patient was made on the basis of the above mentioned typical clinical, EKG, echocardiographic, and CAG features, which corresponded to the typical diagnostic criteria, and the clinical course also corresponded with that of SICM. It is unique in this case that SICM was associated with NPPE due to upper airway obstruction during emergence from anesthesia. Although it is not certain whether NPPE due to upper airway obstruction precipitated SICM or both NPPE and SICM occurred independently and exacerbated the clinical features, it is presumed that excessive catecholamine production due to hypoxia was related to the occurrence of SICM. Also, we could not exclude the possibility that emotional stress caused due to the diagnosis of cancer promoted its occurrence. There is no clear explanation for the individual differences in susceptibility to SICM in spite of a
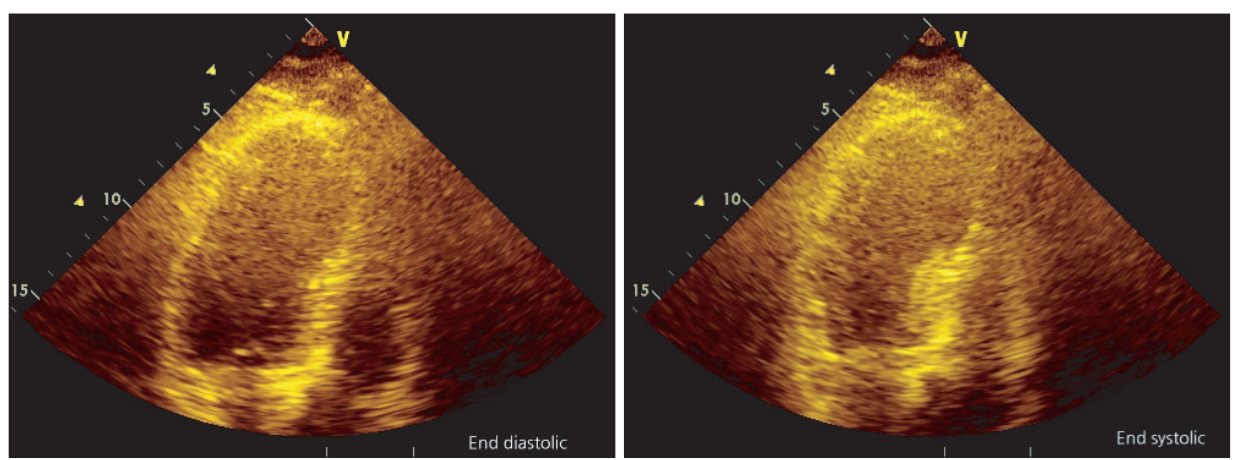

Fig. 3. Transthoracic echocardiography shows left ventricular dysfunction with apical ballooning resembling the octopus trap (takotsubo). 
similar degree of stress or precipitating factors.

When considering the differential diagnosis of acute onset postoperative pulmonary edema, we should take into account both cardiogenic and noncardiogenic pulmonary edema preceded by upper airway obstruction (NPPE), acute lung injury, anaphylaxis, fluid maldistribution, or severe central nervous system trauma (neurogenic pulmonary edema) [15]. The clinical features of this case were most consistent with NPPE due to upper airway obstruction as the most likely cause. Upper airway obstruction leading to NPPE has been reported in the events that include laryngospasm, endotracheal tube occlusion, foreign body aspiration, oropharyngeal surgery or residual curarization [15]. Although according to the clinical features, laryngospasm after extubation is the most likely event related with upper airway obstruction in this case, the possibility of residual curarization cannot be excluded because the duration of anesthesia was relatively short and extubation was performed without confirmation of recovery of neuromuscular function.

There are no uniformly accepted treatment strategies for SICM. The patients with SICM generally might receive supportive management for heart failure and there are some considerations regarding the use of beta adrenergic blockade or inotropes. As with this case, most patients of SICM usually recover fully without complications, but serious complications might occur.

Since during the perioperative period, the emotional or physical stress increases and the patient could be susceptible to SICM, anesthesiologists should become adept at diagnosing SICM and managing these patients properly. Although it is unclear how to manage the patients who have a history of SICM and need to undergo surgery, it would be helpful to try to suppress the production of catecholamines during the perioperative period. However, further research is needed to prevent recurrence of SICM or its management.

\section{References}

1. Wittstein IS. Acute stress cardiomyopathy. Curr Heart Fail Rep 2008; 5: 61-8.
2. Ramakrishna G, Ravi BS, Chandrasekaran K. Apical ballooning syndrome in a postoperative patient with normal microvascular perfusion by myocardial contrast echocardiography. Echocardiography 2005; 22: 606-10.

3. Lentschener C, Vignaux O, Spaulding C, Bonnichon P, Legmann P, Ozier Y. Early postoperative tako-tsubo-like left ventricular dysfunction: transient left ventricular apical ballooning syndrome. Anesth Analg 2006; 103: 580-2.

4. Gavish D, Rozenman Y, Hafner R, Bartov E, Ezri T. Takotsubo cardiomyopathy after general anesthesia for eye surgery. Anesthesiology 2006; 105: 621-3.

5. Consales G, Campiglia L, Michelagnoli G, Gallerani E, Rinaldi S, Del Pace S, et al. Acute left ventricular dysfunction due to Tako-tsubo syndrome after induction of general anesthesia. Minerva Anestesiol 2007; 73: 655-8.

6. Jabaudon M, Bonnin M, Bolandard F, Chanseaume S, Dauphin C, Bazin JE. Takotsubo syndrome during induction of general anaesthesia. Anaesthesia 2007; 62: 519-23.

7. Artukoglu F, Owen A, Hemmerling TM. Tako-Tsubo syndrome in an anaesthetised patient undergoing arthroscopic knee surgery. Ann Card Anaesth 2008; 11: 38-41.

8. Tiwari AK, D'Attellis N. Intraoperative left ventricular apical ballooning: transient takotsubo cardiomyopathy during orthotopic liver transplantation. J Cardiothorac Vasc Anesth 2008; 22: 442-5.

9. Kogan A, Ghosh P, Schwammenthal E, Raanani E. Takotsubo syndrome after cardiac surgery. Ann Thorac Surg 2008; 85: 1439-41.

10. Park JT, Kim JY, Kim YW, Choi KH, Park BH, Lim HK. Stress-induced cardiomyopathy after general anesthesia for total gastrectomy -A case report-. Korean J Anesthesiol 2010; 58: 299-303.

11. Daly MJ, Dixon LJ. Takotsubo cardiomyopathy in two preoperative patients with pain. Anesth Analg 2010; 110: 708-11.

12. Wong AK, Vernick WJ, Wiegers SE, Howell JA, Sinha AC. Preoperative Takotsubo cardiomyopathy identified in the operating room before induction of anesthesia. Anesth Analg 2010; 110: 712-5.

13. Sato H, Tateishi H, Uchida T. Takotsubo-type cardiomyopathy due to multivessel spasm. In: Clinical aspect of myocardial injury: from ischemia to heart failure. Edited by Kodama K, Haze K, Hon M: Tokyo, Kagakuhyouronsha. 1990, pp 56-64.

14. Bybee KA, Prasad A. Stress-related cardiomyopathy syndromes. Circulation 2008; 118: 397-409.

15. Krodel DJ, Bittner EA, Abdulnour R, Brown R, Eikermann M. Case scenario: acute postoperative negative pressure pulmonary edema. Anesthesiology 2010; 113: 200-7. 